\title{
Postpartum-Specific Vital Sign Reference Ranges
}

\author{
Lauren J. Green, BM BSc, Rebecca Pullon, DPhil, Lucy H. Mackillop, MA, Stephen Gerry, MSc, \\ Jacqueline Birks, MSc, Dario Salvi, PhD, Shaun Davidson, PhD, Lise Loerup, PhD, Lionel Tarassenko, DPhil, \\ Jude Mossop, BA(Hons), Clare Edwards, BSc, Rupert Gauntlett, MB ChB, Kate Harding, FRCOG, \\ Lucy C. Chappell, PhD, Marian Knight, DPhil, and Peter J. Watkinson, MD
}

OBJECTIVE: To estimate normal ranges for postpartum maternal vital signs.

METHODS: We conducted a multicenter prospective longitudinal cohort study in the United Kingdom. We recruited women before 20 weeks of gestation without significant comorbidities and with accurately dated singleton pregnancies. Women recorded their own blood pressure, heart rate, oxygen saturation and temperature daily for 2 weeks postpartum. Trained midwives measured participants' vital signs including respiratory rate around postpartum days 1,7 , and 14 .
RESULTS: From August 2012 to September 2016, we screened 4,279 pregnant women; 1,054 met eligibility criteria and chose to take part. Postpartum vital sign data were available for 909 women (86.2\%). Median, or 50th centile (3rd-97th centile), systolic and diastolic blood pressures increased from the day of birth: $116 \mathrm{~mm} \mathrm{Hg}$ (88-147) and $74 \mathrm{~mm} \mathrm{Hg}$ (59-93) to a maximum median of $121 \mathrm{~mm} \mathrm{Hg}(102-143)$ and $79 \mathrm{~mm} \mathrm{Hg}(63-94)$ on days 5 and 6 postpartum, respectively, an increase of $5 \mathrm{~mm} \mathrm{Hg}$ (95\% Cl 3-7) and $5 \mathrm{~mm} \mathrm{Hg}(95 \% \mathrm{Cl} \mathrm{4-6),} \mathrm{respectively.}$ Median (3rd-97th centile) systolic and diastolic blood

From the Nuffield Department of Clinical Neurosciences, the Institute of Biomedical Engineering, Department of Engineering Science, the Nuffield Department of Women's \& Reproductive Health, and the Centre for Statistics in Medicine, University of Oxford, Oxford, United Kingdom; the Department of Anaesthesia, Wellington Hospital, Wellington, New Zealand; and Guy's and St Thomas'NHS Foundation Trust and the Department of Women and Children's Health, King's College, London, and the National Perinatal Epidemiology Unit and the Oxford National Institute for Health Research Biomedical Research Centre, Nuffield Department of Clinical Neurosciences, University of Oxford, Oxford, United Kingdom.

The 4P study is supported by the NIHR Biomedical Research Centre, Oxford and the NIHR Biomedical Research Centre of Guy's and St Thomas' NHS Foundation Trust, London. Lise Loerup was funded by an RCUK Digital Economy Programme (grant number EP/G036861/1, Oxford Centre for Doctoral Training in Healthcare Innovation) and the Clarendon Fund. Stephen Gerry is funded by an NIHR Doctoral Fellowship (DRF-2016-09-073). The funders had no role in study design, dato collection and analysis, decision to publish, or preparation of the manuscript.

The authors thank the significant contribution towards data collection made by research midwives in Oxford (Fenella Roseman), London (Holly Lovell) and Newcastle (Alison Kimber). Fiona Kumar contributed to the study design. The authors would also like to thank Professor Stephen Kennedy and the whole INTERBIO-21st Study team for their generosity in contributing maternal vital sign data to the 4P Study and Mauro Villarroel for help with extracting respiratory rates from photoplethysmography.

Presented at the 43rd Annual Meeting of the MacDonald Obstetric Medicine Society, June 28, 2019, Leeds, United Kingdom.

Each author has confirmed compliance with the journal's requirements for authorship.

Corresponding author: Lauren J. Green, BM, BSc, Nuffeld Department of Clinical Neurosciences, University of Oxford, Oxford, United Kingdom; email: Lauren.Green@ouh.nhs.uk.

Financial Disclosure

Peter J. Watkinson and Lionel Tarassenko, Lucy Chappell and Marian Knight hold grants from the National Institute for Health Research (NIHR). Marian Knight is an NIHR Senior Investigator. Lucy Chappell is an NIHR Research Professor. Lauren J. Green, Lucy H. Mackillop, Lionel Tarassenko, Peter J. Watkinson, Jude Mossop, Clare Edwards, Jacqueline Birks and Dario Salvi receive funding from NIHR Biomedical Research Centre, Oxford. Lucy H. Mackillop works part-time for Sensyne Health. Peter J. Watkinson worked for Sensyne Health. Both have share options in Sensyne Health. Lise Loerup is currently an employee of Boston Consulting Group. Lionel Tarassenko is a nonexecutive Director of Sensyne Health and holds share options in the company, and he is a nonexecutive Director of Oxehealth and holds shares in the company. Sensyne Health markets the System for Electronic Notification and Documentation, which allowes recording of adult vital signs and early warning score calculation. It also markets an application for gestational diabetes management. Oxehealth markets a system to provide contact-free heart and breathing rate monitoring. Neither company had any role in study design, data collection and analysis, decision to publish, or preparation of the manuscript. The other authors did not report any potential conflicts of interest.

(C) 2021 The Author(s). Published by Wolters Kluwer Health, Inc. This is an open-access article distributed under the Creative Commons Attribution License 4.0 $(C C B Y)$, which permits unrestricted use, distribution, and reproduction in any medium, provided the original work is properly cited. ISSN: $0029-7844 / 21$ 
pressure returned to $116 \mathrm{~mm} \mathrm{Hg}(98-137)$ and $75 \mathrm{~mm} \mathrm{Hg}$ (61-91) by day 14 postpartum. The median (3rd-97th centile) heart rate was highest on the day of birth, 84 beats per minute (bpm) (59-110) decreasing to a minimum of $75 \mathrm{bpm}$ (55-101) 14 days postpartum. Oxygen saturation, respiratory rate, and temperature did not change in the 2 weeks postbirth. Median (3rd-97th centile) day-of-birth oxygen saturation was 96\% (93-98). Median (3rd-97th centile) day-of-birth respiratory rate was 15 breaths per minute (10-22). Median (3rd-97th centile) day-of-birth temperature was $36.7^{\circ} \mathrm{C}$ (35.6-37.6).

CONCLUSION: We present widely relevant, postpartum, day-specific reference ranges which may facilitate early detection of abnormal blood pressure, heart rate, respiratory rate, oxygen saturation and temperature during the puerperium. Our findings could inform construction of an evidence-based modified obstetric early warning system to better identify unwell postpartum women.

CLINICAL TRIAL REGISTRATION: ISRCTN, 10838017.

(Obstet Gynecol 2021;137:295-304)

DOI: 10.1097/AOG.0000000000004239

M ost maternal deaths occur postpartum, either within 24 hours of birth $(17 \%$ in the United States $^{1}$; $25 \%$ in the United Kingdom ${ }^{2}$; $50 \%$ worldwide $\left.^{3}\right)$ or in the subsequent 6 weeks $(40 \%$ in the United States, ${ }^{1} 45 \%$ in the United Kingdom ${ }^{2}$ ). The postpartum period often receives less attention than antenatal and intrapartum care. ${ }^{3}$ The leading direct causes of postpartum pregnancy-related mortality are all associated with abnormal vital signs (venous thromboembolism, sepsis, and postpartum hemorrhage in the United States and the United Kingdom ${ }^{1,4}$ and gestational hypertensive disorders worldwide). ${ }^{5}$ Identification of physiologic deterioration postpartum needs improvement, ${ }^{4}$ but normal vital sign ranges are poorly defined. ${ }^{6}$ Previous studies usually report values on one or two occasions often several weeks postpartum. ${ }^{7-14}$ Daily data are rare, often small-scale, and outdated (Walters BNJ, Walters T. Hypertension in the puerperium [letter]. Lancet 1987;2(8554):330. doi: 10.1016/s01406736(87)90912-3). ${ }^{15}$ Fewer data exist for other vital signs postpartum. ${ }^{6}$ Recommended vital signs ranges in national reports (United Kingdom, ${ }^{16}$ Ireland, ${ }^{17}$ United States ${ }^{18}$ ), and modified obstetric early warning scores thresholds ${ }^{19-22}$ are based on expert opinion, with wide national and international variation. Thresholds used during pregnancy are used after birth, without accommodating changes for postpartum maternal physiology. Outside pregnancy, the use of robust estimates of vital sign distributions to generate early warning scores is reliable. ${ }^{23,24}$ The approach may be particularly useful postpartum, where event rates are low. The primary objective of the $4 \mathrm{P}$ (Pregnancy Physiology Pattern Prediction) study was to develop a database of prospective vital sign measurements using standardized measurement techniques throughout pregnancy and the first 2 weeks postpartum. ${ }^{25,26} \mathrm{We}$ included a pragmatic "lowrisk" population, representative of women who would be monitored using a modified obstetric early warning score derived from the normal vital sign ranges obtained. We derived estimates of population distributions and associated centiles from this database.

\section{METHODS}

This work is reported following the STROBE (Strengthening the Reporting of Observational Studies in Epidemiology) guidelines. ${ }^{27} \mathrm{We}$ registered the study (https://doi.org/10.1186/ISRCTN10838017). Detailed methods are published in the protocol. ${ }^{26}$ We conducted a multicenter, longitudinal, observational, cohort study across three U.K. centers. We collected vital sign data during the antenatal, ${ }^{25}$ intrapartum, and postpartum periods. Here we present postpartum data.

Recruitment commenced August 2012 with vital sign collection completed August 2017. The study started in Oxford as a substudy of the INTERBIO21st Fetal Study, approved by Oxford South Central C Research Ethics Committee (REC:08/H0606/ 139), ${ }^{28,29}$ and expanded to include two additional centers (Newcastle and London; with approval granted by South East Coast-Brighton and Sussex Research Ethics Committee REC:14/LO/1312) continuing after completion of INTERBIO-21st (December 2015).

We approached women before 20 weeks of gestation. Eligible women were aged 16 years or older, with a singleton pregnancy, and within category one of the American Society of Anesthesiologists' classification of physical status before pregnancy ("a normal healthy patient without any clinically important comorbidity and without clinically significant past or present medical history"30). Gestational age was determined by ultrasound measurement of crownrump length before 14 weeks of gestation. Full eligibility criteria are in Appendix 1, available online at http://links.lww.com/AOG/C171. Participants provided informed written consent and could withdraw from the study at any time.

The primary outcome for this substudy was postpartum-specific reference ranges comprising vital sign centile distributions for the first 2 weeks of the puerperium. We assessed differences in self- and clinician-taken vital sign measurements. 
We previously found no clinically meaningful differences between first and second measures of vital signs in pregnancy, ${ }^{25}$ so did not collect duplicate readings during the postpartum study period. We previously showed the performance of the devices used to measure each vital sign remained unchanged throughout the study. ${ }^{25}$

Trained research midwives provided instruction in the use of home monitoring equipment to participants around birth, using study standard operating procedures. ${ }^{26}$ Participating women measured and recorded daily vital sign data for four physiologic parameters: blood pressure, heart rate, oxygen saturation, and temperature until day 14 after birth using standardized equipment. Blood pressure was measured with an automated blood pressure monitor validated for use in pregnancy (Microlife 3BT0-A (2)/ WatchBP Home). Heart rate and oxygen saturation were measured with a Bluetooth-enabled pulse oximeter (WristOx2 3150). Temperature was measured with a tympanic thermometer (Genius 2). Additionally, trained research midwives collected vital sign data following study standard operating procedures at home visits on up to three occasions after birth ${ }^{26}$ for five physiologic parameters: blood pressure, heart rate, oxygen saturation, temperature, and respiratory rate. Trained midwives also collected day 0 observations when visiting mothers waiting to be discharged to provide home monitoring equipment. For both participant and midwife-taken vital signs, heart rate, oxygen saturation values, and the photoplethysmography waveform were automatically transmitted via Bluetooth to an Android tablet computer (Samsung Galaxy Tab 4.0), with blood pressure and temperature inputted manually. Midwives measured respiratory rate using two methods: by observing chest wall movement over a 15 -second period and by tapping in time with observed respiratory rate for 1 minute using a software application on the Android tablet computer (October 2015 onward, to explore whether this method was more reliable than the 15 -second method). We also undertook an exploratory analysis to ascertain whether respiratory rate could be extracted from the photoplethysmography waveform recorded by the pulse oximeter.

Participants knew that self-collected vital signs were not reviewed in real time. It remained the participant's responsibility to seek assistance if they felt unwell. Vital sign measurements taken for the study were not included in the clinical record and were not communicated to the clinical team unless blood pressure reached predefined values (systolic higher than $140 \mathrm{~mm} \mathrm{Hg}$ or diastolic higher than
$90 \mathrm{~mm} \mathrm{Hg}$ ) according to study standard operating procedures (Appendix 2, available online at http:// links.lww.com/AOG/C171). Research midwife coordinators performed frequent site visits to carry out midwife training and address any recruitment and equipment issues.

We collected demographic information (age, height, weight, self-reported ethnicity, number of previous pregnancies, smoking status), past medical and obstetric history, current health status, pregnancyrelated health and current medications at the initial assessment. Ethnicity was defined by the participant at the baseline visit according to classification by the National Institute for Health and Care Excellence, as referenced in our protocol. ${ }^{26}$ The option not to give ethnic group information was available to every participant. Ethnicity was collected to allow the generalizability of our population to be considered. At each follow-up appointment, we collected smoking status, current health status, pregnancy-related health and current medications. We extracted medical and obstetric history from the participants' notes.

We published our sample size determination ${ }^{26}$ (and Appendix 3, available online at http://links. lww.com/AOG/C171). In brief, a sample size of 1,000 women would achieve an $\mathrm{SE}$ of $0.05^{*} \mathrm{SD}$ at the 2.5th and 97.5th centiles, and even greater precision at the less extreme centiles. Adequate precision was also met for any subgroup analysis; for example, a sample size of 300 women would achieve an SE of 0. $1 * \mathrm{SD}$ at the 2.5 th and 97.5 th centiles.

We included vital sign data from all participants in the primary analysis (including participants who became lost to follow-up or had missing measures). Once enrolled, we did not exclude women who developed conditions that might affect their vital signs (to generate a pragmatic, representative sample of postpartum women and maximize the clinical applicability of centiles generated). We constructed smoothed centiles for systolic and diastolic blood pressure, heart rate, oxygen saturation, and temperature by day postpartum. We constructed postpartumspecific reference ranges comprising smoothed centiles for vital sign distribution (3rd, 10th, 50th, 90th, and 97th centiles as used by the World Health Organization Multicentre Growth Reference Study, ${ }^{31,32}$ with corresponding 95\% CIs) for all women. We followed the statistical methods used in the INTERGROWTH-21st Project for fetal growth ${ }^{33-36}$ to generate postpartum day-specific centiles. We explored different statistical methods to achieve the best fit to the data (see Appendix 3, http://links.lww. $\mathrm{com} / \mathrm{AOG} / \mathrm{C} 171)$. As respiratory rate was only 
recorded at midwife visits (meaning sufficient data for smoothed centiles would not be expected), we constructed empirical centiles based on grouping the data into four periods of 3.5 days, with results plotted at the mid-point of each period.

Where both self- and clinician-taken vital sign measurements were recorded within an hour, we assessed agreement using the Bland-Altman method. We pooled data when there was no substantial bias and limits of agreement were adequate. Where two sets of vital sign measurements were recorded by the same method (usually two sets of self-taken measurements) within an hour, we used the mean of the two values in our analyses.

We conducted a predefined subgroup analysis of the effect of parity on the postpartum reference ranges. To explore whether limiting the population to those of optimal health would affect results, we defined a "restrictive" population of women aged younger than 40 years with body mass indexes (BMIs, calculated as weight in kilograms divided by height in meters squared) between 18.5 and 29.9 who did not smoke and did not have comorbidities. In this "restrictive" population, we excluded measures from women who developed a severe maternal condition during pregnancy (severe preeclampsia; hemolysis, elevated liver enzymes, and low platelet count [HELLP] syndrome; or eclampsia, as defined in the study proto$\mathrm{col}^{26}$ ), in line with previous work. ${ }^{37}$ We conducted a post hoc analysis using these definitions to compare with the analysis of the full "pragmatic" population. We also undertook post hoc analyses of the effect of epidural and epidural or anesthesia on postpartum reference ranges.

\section{RESULTS}

We screened 4,279 women between August 1, 2012, and December 28, 2016, of whom 1,054 agreed to take part. A cohort of 909 women contributed postpartum vital sign data (Fig. 1). Study cohort demographic characteristics were similar across sites (Table 1). At the first antenatal visit mean (SD) gestational age was 13.2 (2.5) weeks; maternal age 32.2 (4.7) years; BMI 24.8 (4.7) and 44.9\% (408/909) were nulliparous. Maternal characteristics were similar in the postpartum cohort to those of all women enrolled (Appendix 4, available online at http://links.lww. com/AOG/C171). ${ }^{25}$

The median number of times per woman where vital signs were recorded was 11 (interquartile range 8 to 13). In total, 9,621 sets of vital sign data were recorded. Of these, blood pressure was recorded nearly always $(9,534 / 9,621,99 \%)$, heart rate $96 \%$

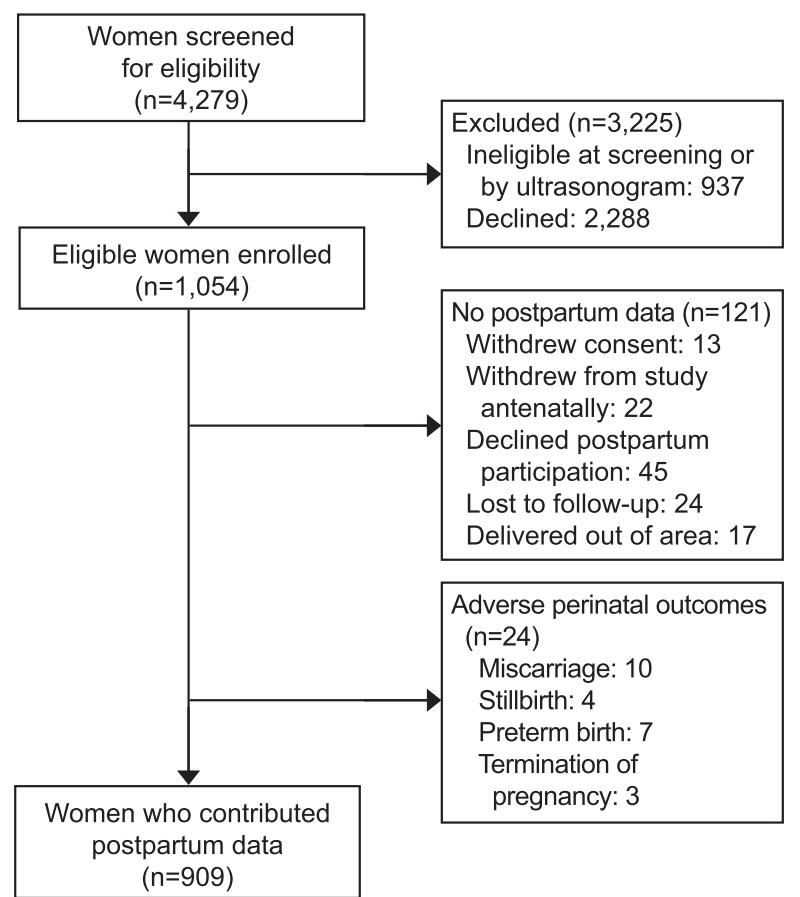

Fig. 1. Flowchart of participants in the study.

Green. Postpartum Vital Sign Reference Ranges. Obstet Gynecol 2021.

$(9,221 / 9,621)$, oxygen saturation $96 \%(9,224 / 9,621)$ and temperature $97 \%(9,347 / 9,621)$. Respiratory rate was recorded at $92 \%$ of midwife visits $(1,535 / 1,600$ visits up to day 14). An abnormal blood pressure recording necessitating referral to the woman's usual clinical team occurred at $0.03 \%$ of visits $(3 / 9,621$ observations; 3/909 women). Table 2 details pregnancy outcomes and perinatal events within the study cohort.

The Bland-Altman plots did not show significant bias between self- and clinician-taken vital sign recordings (Appendix 5, available online at http:// links.lww.com/AOG/C171). Therefore, we pooled data from the two groups for systolic and diastolic blood pressure, heart rate, oxygen saturation and temperature. Systolic blood pressure increased from the day of birth (day 0$)$, from a median, or 50th centile, of $116 \mathrm{~mm} \mathrm{Hg}$ (3rd-97th centile 88-147) to a maximum median of $121 \mathrm{~mm} \mathrm{Hg}$ (3rd-97th centile 102-143) 5.4 days postpartum, an increase of $5 \mathrm{~mm} \mathrm{Hg}(95 \%$ CI $3-$ 7). Systolic blood pressure then decreased to a nadir of median $116 \mathrm{~mm} \mathrm{Hg}$ (3rd-97th centile 98-137) 14 days postpartum, a difference of $-6 \mathrm{~mm} \mathrm{Hg}(95 \% \mathrm{CI}$ -6 to -5 ) from maximum to minimum systolic blood pressure. Diastolic blood pressure was lowest on the day of birth: median, or 50th centile, $74 \mathrm{~mm} \mathrm{Hg}$ (3rd to 97 th centile 59-93). Diastolic blood pressure rose 
Table 1. Baseline Maternal Characteristics

\begin{tabular}{|c|c|c|c|c|}
\hline Characteristic & Oxford [n=687 (76)] & London $[n=86(10)]$ & Newcastle [n=136 (15)] & Total $(\mathrm{N}=909)$ \\
\hline Age (y) & $32.1 \pm 4.7$ & $34.0 \pm 4.2$ & $31.5 \pm 5.0$ & $32.2 \pm 4.7$ \\
\hline Weight (kg) & $67.2 \pm 12.7$ & $69.3 \pm 15.8$ & $70.2 \pm 16.3$ & $67.9 \pm 13.7$ \\
\hline BMI $\left(\mathrm{kg} / \mathrm{m}^{2}\right)$ & $24.5 \pm 4.2$ & $25.3 \pm 5.4$ & $25.8 \pm 5.8$ & $24.8 \pm 4.7$ \\
\hline \multicolumn{5}{|l|}{ BMI category $\left(\mathrm{kg} / \mathrm{m}^{2}\right)$} \\
\hline Normal weight (18.5-24.9) & $421(61.3)$ & $53(61.6)$ & $72(53.7)$ & $546(60.2)$ \\
\hline Overweight (25.0-29.9) & $188(27.4)$ & $17(19.8)$ & $34(25.4)$ & $239(26.4)$ \\
\hline Obese (30 or higher) & $78(11.4)$ & $16(18.6)$ & $28(20.9)$ & $122(13.5)$ \\
\hline Nulliparous & $300(43.7)$ & $48(55.8)$ & $60(44.1)$ & $408(44.9)$ \\
\hline \multicolumn{5}{|l|}{ Ethnicity } \\
\hline White & $591(86.0)$ & $60(69.8)$ & $132(97.1)$ & $783(86.1)$ \\
\hline Asian & $26(3.8)$ & $11(12.8)$ & $3(2.2)$ & $40(4.4)$ \\
\hline African or Caribbean & $4(0.6)$ & $12(14.0)$ & $0(0)$ & $16(1.8)$ \\
\hline Mixed & $13(1.9)$ & $2(2.3)$ & $1(0.7)$ & $16(1.8)$ \\
\hline Other & $53(7.7)$ & $1(1.2)$ & $0(0)$ & $54(5.9)$ \\
\hline Smoker & $45(6.6)$ & $3(3.5)$ & $8(5.9)$ & $56(6.2)$ \\
\hline Anemia* & $2(0.3)$ & $3(3.5)$ & $1(0.7)$ & $6(0.7)$ \\
\hline Pregestational diabetes & $2(0.3)$ & $1(1.2)$ & $3(2.2)$ & $6(0.7)$ \\
\hline Pre-existing hypertension ${ }^{+}$ & $15(2.2)$ & $0(0)$ & $0(0)$ & $15(1.7)$ \\
\hline Cardiac disease $^{\ddagger}$ & $10(1.5)$ & $1(1.2)$ & $0(0)$ & $11(1.2)$ \\
\hline Pre-existing renal disease & $14(2)$ & $1(1.2)$ & $4(2.9)$ & $19(2.1)$ \\
\hline
\end{tabular}

Data are mean \pm SD or $\mathrm{n}(\%)$.

BMI, body mass index

Data on BMI were missing for two women.

Ethnicity was defined by the participant at baseline visit according to classification by the National Institute for Health and Care Excellence, as referenced in our protocol. ${ }^{26}$ The option not to give ethnic group information was available to every participant. Ethnicity was collected to allow the generalizability of our population to be considered.

* Defined as hemoglobin less than $110 \mathrm{~g} / \mathrm{L}$.

+ Unmedicated.

${ }^{\ddagger}$ Nonischemic noncongenital.

to a maximum median of $79 \mathrm{~mm} \mathrm{Hg}$ (3rd-97th centile 63-94) 6 days postpartum, a difference of $5 \mathrm{~mm} \mathrm{Hg}$ (95\% CI 4-6) from minimum to maximum diastolic blood pressure. Diastolic blood pressure subsequently decreased: median $75 \mathrm{~mm} \mathrm{Hg}$ (3rd-97th centile 6090) 14 days postpartum, a change of $-4 \mathrm{~mm} \mathrm{Hg}(95 \%$
CI -5 to -3 ) (Fig. 2). The median, or 50th centile, heart rate was highest on the day of birth: 84 beats per minute (bpm) (3rd-97th centile 59-110). Median heart rate decreased progressively to the 7 th day after birth: day 7 median $76 \mathrm{bpm}$ (3rd-97th centile 54-101) (Fig. 3), a difference of $-8 \mathrm{bpm}(95 \% \mathrm{CI}-11$ to -5$)$.

Table 2. Pregnancy Complications and Perinatal Outcomes

\begin{tabular}{|c|c|c|c|c|}
\hline Pregnancy Complications and Birth Outcomes & $\begin{array}{c}\text { Oxford } \\
{[n=687(76)]}\end{array}$ & $\begin{array}{c}\text { London }[\mathrm{n}=86 \\
(10)]\end{array}$ & $\begin{array}{c}\text { Newcastle } \\
{[n=136(15)]}\end{array}$ & $\begin{array}{c}\text { Total } \\
(\mathrm{N}=909)\end{array}$ \\
\hline Gestational diabetes mellitus & $25(3.6)$ & $4(4.7)$ & $6(4.7)$ & $35(3.9)$ \\
\hline Gestational hypertension & $43(6.3)$ & $1(1.2)$ & $1(0.8)$ & $45(5.0)$ \\
\hline Preeclampsia & $9(1.3)$ & $0(0)$ & $7(5.4)$ & $16(1.8)$ \\
\hline Severe preeclampsia, HELLP syndrome, eclampsia & $0(0)$ & $0(0)$ & $3(2.3)$ & $3(0.3)$ \\
\hline Preterm birth at less than $370 / 7$ wk & $37(5.4)$ & $3(3.5)$ & $9(6.6)$ & $49(5.4)$ \\
\hline Spontaneous vaginal birth & $436(63.5)$ & $49(57.0)$ & $86(65.6)$ & $571(63.2)$ \\
\hline Assisted vaginal birth & $126(18.3)$ & $12(14.0)$ & $10(7.6)$ & $148(16.4)$ \\
\hline Cesarean birth & $125(18.2)$ & $25(29.1)$ & $35(26.7)$ & $185(20.5)$ \\
\hline Birth weight at $370 / 7 \mathrm{wk}$ of gestation or later (g) & $3,351 \pm 581$ & $3,423 \pm 594$ & $3,382 \pm 638$ & $3,363 \pm 591$ \\
\hline $\begin{array}{l}\text { Term low birth weight (less than 2,500 g, } 370 / 7 \mathrm{wk} \text { of } \\
\text { gestation or later) }\end{array}$ & $16 / 647(2.5)$ & $1 / 83(1.2)$ & $3 / 122(2.5)$ & $20 / 852(2.3)$ \\
\hline
\end{tabular}

Data are $\mathrm{n}(\%)$ or mean \pm SD.

HELLP, hemolysis, elevated liver enzymes, and low platelet count.

Mode of birth missing (delivered out of area) for five women. 




Fig. 2. Smoothed centiles for systolic blood pressure (upper line and centiles) and diastolic blood pressure (lower line and centiles) in $\mathrm{mm} \mathrm{Hg}$. Day 0 indicates day of birth.

Green. Postpartum Vital Sign Reference Ranges. Obstet Gynecol 2021.

There was no further significant change in heart rate by day 14 , with a median value of $75 \mathrm{bpm}$ (3rd-97th centile $55-101)$, a difference of $-0.5 \mathrm{bpm}(95 \%$ CI -1.9 to 0.8$)$.

Respiratory rate remained unchanged through the postpartum period from the median day-of-birth respiratory rate of 15 breaths per minute (3rd-97th centile 10-22) (Fig. 4). Grouped-day postpartum-specific values for respiratory rate centiles (from tapping method), with the methodology and centiles for respiratory rate derived from the photoplethysmography waveform, are in Appendix 6, available online at http://links. lww.com/AOG/C171. Median, or 50th centile, oxygen saturation was $96 \%$ (3rd-97th centile 93-98) on the day of birth, with no clinically significant difference to day 14 (Fig. 5). Median, or 50th centile, temperature did not change from the day-of-birth value of $36.7^{\circ} \mathrm{C}(3 \mathrm{rd}-$ 97th centile 35.6-37.6) (Fig. 6).

For each vital sign measured, postpartum-specific values for the smoothed centiles and a smoothed day postpartum-specific centile plot with associated 95\% CI can be found in Appendix 6 (http://links.lww.com/ AOG/C171). Applying the "restrictive" population definitions reduced the cohort to 550 of 909 women (Appendix 7, available online at http://links.lww.com/ AOG/C171). There were no clinically significant differences in vital sign reference ranges between the pragmatic and restrictive populations. Although sys-

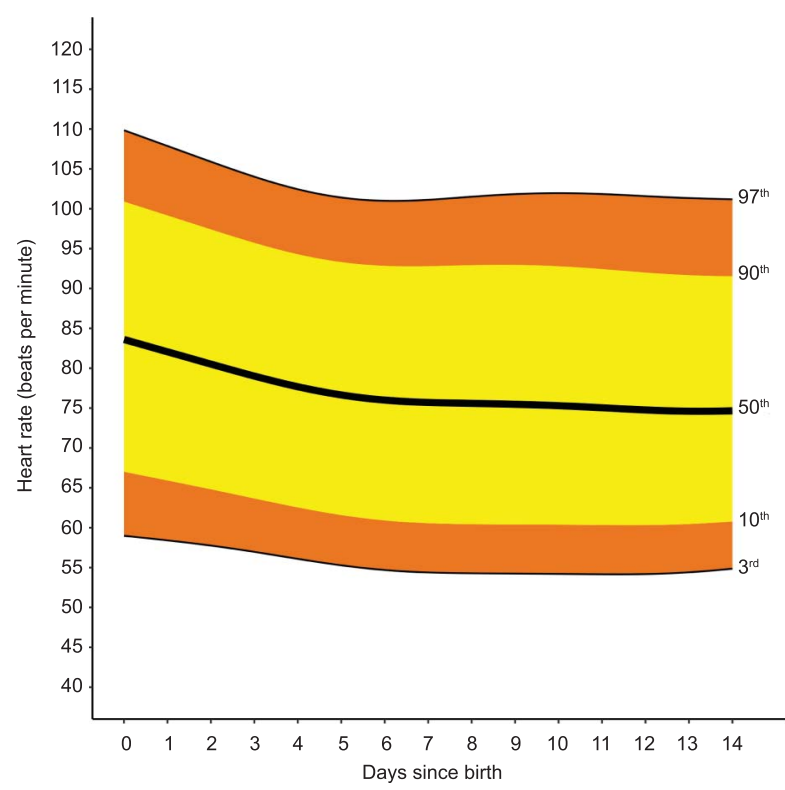

Fig. 3. Smoothed centiles for heart rate in beats per minute. Day 0 indicates day of birth.

Green. Postpartum Vital Sign Reference Ranges. Obstet Gynecol 2021.

tolic and diastolic blood pressure centiles were numerically slightly lower in the restrictive cohort (higher blood pressures having been excluded) the relatively narrow CIs mainly overlapped.

Median heart rates from 408 nulliparous women were mainly 3-6 bpm higher (with relatively tight CIs

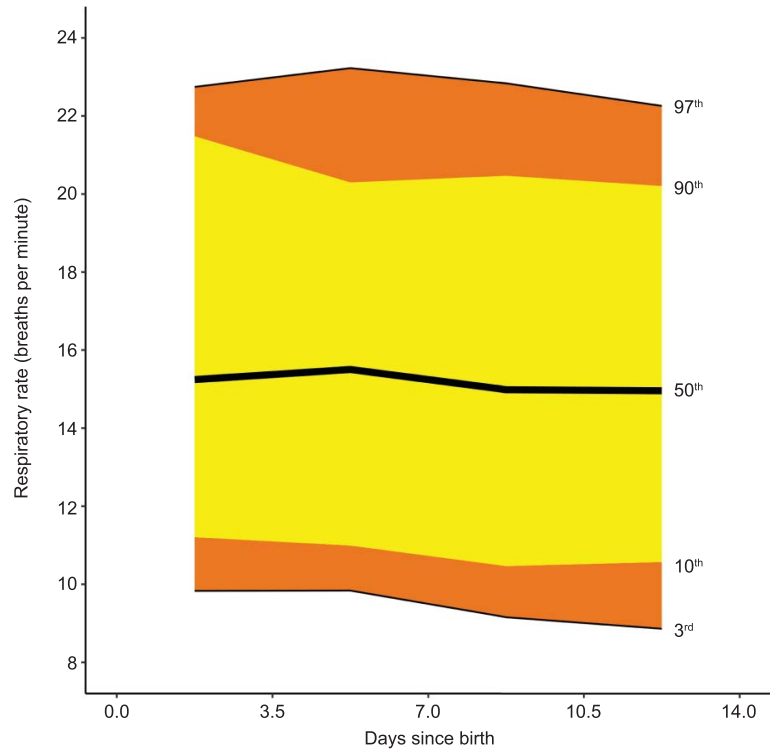

Fig. 4. Smoothed centiles for respiratory rate (breaths per minute). Day 0 indicates day of birth.

Green. Postpartum Vital Sign Reference Ranges. Obstet Gynecol 2021. 




Fig. 5. Smoothed centiles for oxygen saturation (\%). Day 0 indicates day of birth.

Green. Postpartum Vital Sign Reference Ranges. Obstet Gynecol 2021

that did not overlap) than those from the 501 parous women (Appendix 8, available online at http://links. lww.com/AOG/C171). There were no differences between nulliparous and parous women in other vital signs.

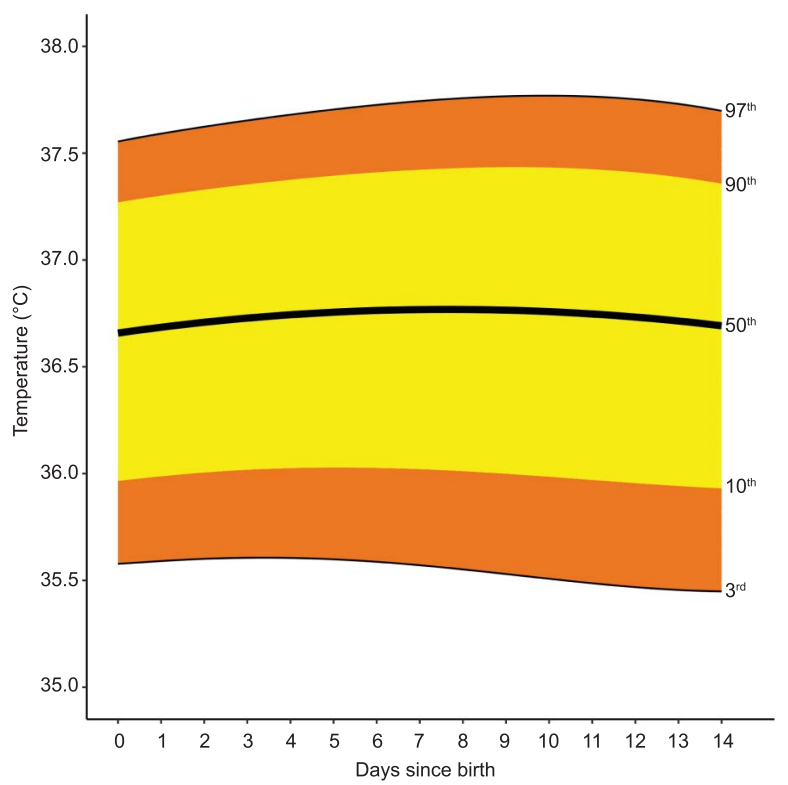

Fig. 6. Smoothed centiles for temperature $\left({ }^{\circ} \mathrm{C}\right)$. Day 0 indicates day of birth.

Green. Postpartum Vital Sign Reference Ranges. Obstet Gynecol 2021.
Median heart rates were around $2 \mathrm{bpm}$ higher (with CIs that did not overlap) in the 226 women who received epidural analgesia in the second week after birth compared with those who did not (683 women, Appendix 9, available online at http://links.lww.com/ AOG/C171). Similarly, median heart rates were 3-4 bpm higher in the 429 women who received either an epidural or a general anesthetic throughout the 2 postpartum weeks in comparison with those who did not (480 women, Appendix 10 [Appendix 10 is available online at http://links.lww.com/AOG/C171]). There were no differences in other vital signs.

\section{DISCUSSION}

Our multicenter study includes longitudinal data from more than 900 women from whom we produced evidence-based postpartum day-specific centiles for vital signs for the 2 weeks after birth. Blood pressure rose after birth until around days 5-6. The rises in median systolic and diastolic blood pressure are similar to those found in small studies from more than 30 years ago (Walters et al. Lancet 1987;2(8554):330. doi: $10.1016 / \mathrm{s} 0140-6736(87) 90912-3) .{ }^{15,38}$ These are suggested to result from mobilization of extracellular fluid and sodium accumulated during pregnancy. ${ }^{39}$ However, these studies did not describe the reference ranges associated with the rise. After the day of birth, the 3rd centile for systolic blood pressure was never less than $97 \mathrm{~mm} \mathrm{Hg}$ in the full or restrictive populations. This is substantially higher than the $90 \mathrm{~mm} \mathrm{Hg}$ systolic blood pressure threshold used to recognize sepsis in and after pregnancy ${ }^{40,41}$ and the trigger for escalation in modified obstetric early warning score charts. ${ }^{16-18,42}$ Whether increasing the threshold could improve earlier detection of deteriorating women requires investigation. In our full population, more than $3 \%$ of women had systolic blood pressures higher than $140 \mathrm{~mm} \mathrm{Hg}$ between days 3 and 8 . In both the full and restrictive populations, the 97th centile for diastolic blood pressure was higher than $90 \mathrm{~mm} \mathrm{Hg}$ on days 2-9 postbirth.

These values are higher than the recommended diagnostic or treatment threshold of higher than 140/ $90 \mathrm{~mm} \mathrm{Hg}$ as recommended by the International Society for the Study of Hypertension in Pregnancy. ${ }^{43}$ Our normative values are for a single measurement of blood pressure, as we showed previously there are no clinically meaningful population differences between first and second measures of vital signs in pregnancy. ${ }^{25}$ Though high readings would be repeated before making a diagnosis of hypertension, our findings support the higher postpartum treatment thresholds of $150 / 100 \mathrm{~mm} \mathrm{Hg}$ or higher recommended by the U.K. National Institute for Health and Care Excellence. ${ }^{4}$ 
In our previous systematic review, we could not find reliable estimations of the outer centiles of heart rate postpartum. ${ }^{6}$ In our previous work with the $4 \mathrm{P}$ cohort, heart rates higher than $100 \mathrm{bpm}$ occurred in more than $10 \%$ of women at 40 weeks of gestation. ${ }^{25}$ Postpartum heart rates of greater than $100 \mathrm{bpm}$ were much less common, with the 90th centile for heart rate $100 \mathrm{bpm}$ or less from day 1 postpartum. The 97 th centile decreased from $110 \mathrm{bpm}$ on the day of birth to $102 \mathrm{bpm}$ by day 7 . These findings suggest that thresholds of greater than 100-120 bpm as a moderate-risk threshold for modified obstetric early warning score escalation ${ }^{16,17,42}$ may be too wide postpartum.

Postpartum thresholds for respiratory rates are poorly described. The median day-of-birth respiratory rate of 15 (3rd-97th centile 10-22) breaths per minute is unchanged from the rate seen in the antenatal $4 \mathrm{P}$ cohort. ${ }^{25}$ As during pregnancy, our work shows that a respiratory rate of greater than 22 breaths per minute (as used in the qSOFA [quick Sepsis Related Organ Failure Assessment] tool ${ }^{45}$ ) would occur in fewer than 3\% of observations postnatally, suggesting that this threshold could reasonably translate from other medical practice. Current moderate-risk and high-risk thresholds for respiratory rate of 21-24 and 25 breaths per minute or more (as advocated by the U.K. Sepsis Trust, ${ }^{41}$ IMEWS $^{17}$ and the Scottish Patient Safety Programme ${ }^{42}$ ) may more accurately identify women at risk of sepsis than moderate-risk $^{16}$ and high-risk ${ }^{16,18}$ thresholds of 21-30 and more than 30 breaths per minute.

The median postpartum oxygen saturation is persistently close to $96 \%$, consistent with the end of pregnancy in the $4 \mathrm{P}$ cohort. ${ }^{25}$ As during pregnancy, oxygen saturation less than $93 \%$ is an abnormal finding after birth. Because $94 \%$ is around the 10th centile throughout the 14 days postpartum, alerting thresholds currently in use of less than $95 \% 0^{16-18}$ or $94 \%$ or less ${ }^{42}$ will commonly cause alerts in healthy women.

There was no clinically significant temperature variation postpartum, with median temperatures very similar to those found during pregnancy. ${ }^{25}$ However, the 97 th centile was up to $37.8^{\circ} \mathrm{C}$ postpartum rather than $37.5^{\circ} \mathrm{C}$ in pregnancy, still lower than the high-risk threshold for escalation of $38^{\circ} \mathrm{C}$ or higher ${ }^{16,17,42}$ used in many modified obstetric early warning scores. As in pregnancy, the lower 10 th centile for temperature lies between $35.9^{\circ} \mathrm{C}$ and $36.0^{\circ} \mathrm{C}$. The lower limit moderaterisk escalation thresholds of less than $36^{\circ} \mathrm{C}$ suggested for sepsis ${ }^{41}$ would therefore cause an alert to be raised in around $10 \%$ of healthy women.

Although higher blood pressures were excluded from the restrictive cohort, blood pressure differences from the pragmatic cohort were marginal. In the nulliparous, epidural, and anesthesia subgroups, heart rates were marginally higher than in the respective comparator cohorts. All other vital sign centiles were similar between comparator subgroups. From these data, it seems unlikely that different alert thresholds for vital signs would be required for any particular subgroup in clinical practice.

The 4P study had secondary objectives of developing a centile-based early warning score and investigating new patterns in vital sign data that will be explored in further work. Our study population is of similar age to the U.K. national average for pregnancy ${ }^{46}$ and with similar BMIs as previous U.K. findings. ${ }^{47}$ Women were predominantly of White European ethnicity $(85.2 \%)$, equivalent to the most recent England and Wales census data $\left.(86 \%)^{48}\right)$. Our population therefore appears representative and applicable to clinical practice. Extending to an international population would improve external validity for other settings. By using standardized pregnancy-specific equipment to collect data prospectively from three sites, we are confident our reference ranges are robust.

We present centiles as recommended by the World Health Organization, from a healthy but not overly selected pregnant cohort to provide reliable data for clinicians in clinical practice. These reference ranges can facilitate earlier recognition of unwell postpartum women to reduce morbidity and ultimately minimize maternal mortality. In conjunction with our published antenata $^{25}$ and planned intrapartum reference ranges, our study will help inform construction of a timespecific modified obstetric early warning score system for use throughout pregnancy and the puerperium.

\section{REFERENCES}

1. Creanga AA, Syverson C, Seed K, Callaghan WM, Author OG Pregnancy-related mortality in the United States, 2011-2013 HHS public access author manuscript. Obstet Gynecol 2017; 130:366-73. doi: 10.1097/AOG.0000000000002114

2. Knight M, Bunch K, Tuffnell D, Shakespeare J, Kotnis R, Kenyon S, et al. Saving lives, improving mothers' care. Lessons learned to inform maternity care from the UK and Ireland Confidential Enquiries into Maternal Deaths and Morbidity 2015-17. Accessed December 11, 2020. https://www.npeu.ox. ac.uk/assets/downloads/mbrrace-uk/reports/MBRRACE-UK $\% 20$ Maternal $\% 20$ Report $\% 202019 \% 20-\% 20$ WEB $\% 20$ VERSION.pdf

3. Matthews M, Von Xylander S, Jelka Z. WHO technical consultation on postpartum and postnatal care. Accessed December 11, 2020. https://apps.who.int/iris/bitstream/handle/10665/70432/WHO_MPS_10.03_eng.pdf;jsessionid=A7AAB21B67476A 93 D95E70A $\overline{3}$ CA91DD1C?sequence $=1$

4. Knight M, Buch K, Tuffnell D, Jayakody H, Shakespeare J, Kotnis R, et al. Saving lives, improving mothers' care. Lessons learned to inform maternity care from the UK and Ireland Confidential Enquiries into Maternal Deaths and Morbidity 
2014-16. National Perinatal Epidemiology Unit, University of Oxford; 2018.

5. Say L, Chou D, Gemmill A, Tunçalp Ö, Moller AB, Daniels J, et al. Global causes of maternal death: a WHO systematic analysis. Lancet Glob Heal 2014;2:e323-33. doi: 10.1016/S2214109X(14)70227-X

6. Loerup L, Pullon RM, Birks J, Fleming S, Mackillop LH, Gerry $\mathrm{S}$, et al. Trends of blood pressure and heart rate in normal pregnancies: a systematic review and meta-analysis. BMC Med 2019;17:167. doi: 10.1186/s12916-019-1399-1

7. Halligan A, O'Brien E, O'Malley K, Mee F, Atkins N, Conroy $\mathrm{R}$, et al. Twenty-four-hour ambulatory blood pressure measurement in a primigravid population. J Hypertens 1993;11:869-73. doi: 10.1097/00004872-199308000-00014

8. Blake MJ, Martin A, Manktelow BN, Armstrong C, Halligan AW, Panerai RB, et al. Changes in baroreceptor sensitivity for heart rate during normotensive pregnancy and the puerperium. Clin Sci 2000;98:259-68.

9. O’Donaghue K. Physiological changes in pregnancy. In: Baker P, Kenny L, editors. Obstetrics by ten teachers. 19th ed. CRC Press; 2011. p. 319.

10. Lo C, Taylor RS, Gamble G, McCowan L, North RA. Use of automated home blood pressure monitoring in pregnancy: is it safe? Am J Obstet Gynecol 2002;187:1321-8. doi: 10. 1067/mob.2002.126847

11. Churchill D, Beevers DG. Differences between office and 24hour ambulatory blood pressure measurement during pregnancy. Obstet Gynecol 1996;88:455-61. doi: 10.1016/00297844(96)00192-5

12. Mahendru AA, Everett TR, Wilkinson IB, Lees CC, McEniery CM. A longitudinal study of maternal cardiovascular function from preconception to the postpartum period. J Hypertens 2014;32:849-56. doi: 10.1097/HJH.0000000000000090

13. Rang S, van Montfrans GA, Wolf H. Serial hemodynamic measurement in normal pregnancy, preeclampsia, and intrauterine growth restriction. Am J Obstet Gynecol 2008;198:519.e1-9.

14. Grindheim G, Estensen ME, Langesaeter E, Rosseland LA, Toska K. Changes in blood pressure during healthy pregnancy: a longitudinal cohort study. J Hypertens 2012;30:342-50. doi: 10.1097/HJH.0b013e32834f0b1c

15. Walters BNJ, Thompson ME, Lee A, De Swiet M. Blood pressure in the puerperium. Clin Sci 1986;71:589-94. doi: 10 . 1042/cs0710589

16. Lewis G, editor. Saving mothers' lives. Reviewing maternal deaths to make motherhood safer - 2003-2005: the seventh report of the confidential enquiries into maternal deaths in the United Kingdom. CRC Press; 2007.

17. Department of Health. Irish maternity early warning system (IMEWS) V2 (NCEC national clinical guideline No.4 version 2). Accessed Oct 8, 2020. https://www.gov.ie/en/collection/ 517f60-irish-maternity-early-warning-system-imews-version-2/

18. Mhyre JM, D'Oria R, Hameed AB, Lappen JR, Holley SL, Hunter $\mathrm{SK}$, et al. The maternal early warning criteria: a proposal from the national partnership for maternal safety. Obs Gynecol 2014;124: 782-6. doi: 10.1097/AOG.0000000000000480

19. McGlennan AP, Sherratt K. Charting change on the labour ward. Anaesthesia 2013;68:338-41. doi: 10.1111/anae.12189

20. Smith GB, Isaacs R, Andrews L, Wee MYK, van Teijlingen E, Bick DE, et al. Vital signs and other observations used to detect deterioration in pregnant women: an analysis of vital sign charts in consultant-led UK maternity units Obstetric vital signs charts. Int J Obstet Anesth 2017;30:44-51. doi: 10.1016/j.ijoa. 2017.03.002
21. Isaacs RA, Wee MYK, Bick DE, Beake S, Sheppard ZA, Thomas S, et al. A national survey of obstetric early warning systems in the United Kingdom: five years on. Anaesthesia 2014;69:687-92. doi: 10.1111/anae.12708

22. Friedman AM. Maternal early warning systems. Obstet Gynecol Clin North Am 2015;42:289-98. doi: 10.1016/j.ogc.2015. 01.006

23. Tarassenko L, Clifton DA, Pinsky MR, Hravnak MT, Woods JR, Watkinson PJ. Centile-based early warning scores derived from statistical distributions of vital signs. Resuscitation 2011; 82:1013-8. doi: 10.1016/j.resuscitation.2011.03.006

24. Watkinson PJ, Pimentel MAF, Clifton DA, Tarassenko L. Manual centile-based early warning scores derived from statistical distributions of observational vital-sign data. Resuscitation 2018;129:55-60. doi: 10.1016/j.resuscitation.2018.06.003

25. Green LJ, Mackillop LH, Salvi D, Pullon RM, Loerup L, Tarassenko L, et al. Gestation-specific vital sign reference ranges in pregnancy. Obs Gynecol 2020;135:653-64. doi: 10 . 1097/AOG.0000000000003721

26. Kumar F, Kemp J, Edwards C, Pullon RM, Loerup L, Triantafyllidis A, et al. Pregnancy Physiology Pattern Prediction study (4P study): protocol of an observational cohort study collecting vital sign information to inform the development of an accurate centile-based obstetric early warning score. BMJ Open 2017;7: e016034. doi: 10.1136/bmjopen-2017-016034

27. von Elm E, Altman DG, Egger M, Pocock SJ, Gøtzsche PC, Vandenbroucke JP. The strengthening the reporting of observational studies in epidemiology (STROBE) statement: guidelines for reporting observational studies. Int J Surg 2014;12: 1495-9. doi: 10.1016/j.ijsu.2014.07.013

28. The INTERBIO-21st Consortium. INTERBIO-21st study protocol. Accessed Oct 8, 2020. www.interbio21.org.uk

29. Stirnemann J, Villar J, Salomon LJ, Ohuma E, Ruyan P, Altman DG, et al. International estimated fetal weight standards of the INTERGROWTH-21stProject. Ultrasound Obstet Gynecol 2017;49:478-86. doi: 10.1002/uog. 17347

30. American Society of Anesthesiologists. ASA physical status classification system. Accessed Oct 8, 2020. https://www. asahq.org/standards-and-guidelines/asa-physical-status-classification-system

31. De Onis M. WHO child growth standards based on length/height, weight and age. Acta Paediatr Int J Paediatr 2006; 95(suppl 450):1-101. doi: 10.1111/j.1651-2227.2006.tb02378.x

32. WHO Multicentre Growth Reference Study Group, de Onis M, Onyango A, Borghi E, Siyam A, Pinol A. WHO child growth standards: growth velocity based on weight, length and head circumference: methods and development. Accessed Oct 8, 2020. https://apps.who.int/iris/handle/10665/44026

33. Papageorghiou AT, Kennedy SH, Salomon LJ, Ohuma EO, Ismail LC, Barros FC, et al. International standards for early fetal size and pregnancy dating based on ultrasound measurement of crown-rump length in the first trimester of pregnancy. Ultrasound Obstet Gynecol 2014;44:641-8. doi: 10.1002/uog. 13448

34. Altman DG, Ohuma EO. Statistical considerations for the development of prescriptive fetal and newborn growth standards in the INTERGROWTH-21stProject. BJOG Int J Obstet Gynaecol 2013;120(suppl 2):71-6. doi: 10.1111/1471-0528. 12031

35. Ohuma EO, Altman DG. The INTERGROWTH-21st Project. Statistical methodology for constructing gestational age-related charts using cross-sectional and longitudinal data: the INTERGROWTH-21st project as a case study. Stat Med 2019;38:3507-26. doi: 10.1002/sim.8018 
36. Ohuma EO, Altman DG. The INTERGROWTH-21st Project. Design and other methodological considerations for the construction of human fetal and neonatal size and growth charts. Stat Med 2019;38:3527-39. doi: 10.1002/sim.8000

37. Villar J, Papageorghiou AT, Pang R, Ohuma EO, Ismail LC, Barros FC, et al. The likeness of fetal growth and newborn size across non-isolated populations in the INTERGROWTH-21st project: the fetal growth longitudinal study and newborn crosssectional study. Lancet Diabetes Endocrinol 2014;2:781-92. doi: 10.1016/S2213-8587(14)70121-4

38. Atkins AFJ, Watt JM, Milan P, Davies P, Crawford JS. A longitudinal study of cardiovascular dynamic changes throughout pregnancy. Eur J Obstet Gynecol Reprod Biol 1981;12:215-24. doi: 10.1016/0028-2243(81)90012-5

39. Magee L, von Dadelszen P. Prevention and treatment of postpartum hypertension. The Cochrane Database of Systematic Reviews 2013, Issue 4. Art. No.: CD004351. doi: 10. 1002/14651858.CD004351.pub3

40. Acosta CD, Kurinczuk JJ, Lucas DN, Tuffnell DJ, Sellers S, Knight M. Severe maternal sepsis in the UK, 2011-2012: a national case-control study. PLoS Med 2014;11:2011-2. doi: 10.1371/journal.pmed.1001672

41. UK Sepsis Trust. Inpatient maternal sepsis tool. Accessed October 8, 2020. https://sepsistrust.org/professional-resources/clinical/

42. Scottish Patient Safety Programme, Maternity and Children Quality Improvement Collaborative. Scottish maternity early warning score (MEWS) chart. Accessed Oct 8, 2020. https:// ihub.scot/media/5308/national-mews-chart_web.pdf

43. Brown MA, Magee LA, Kenny LC, Karumanchi SA, McCarthy FP, Saito S, et al. Hypertensive disorders of pregnancy: ISSHP classification, diagnosis, and management recommendations for international practice. Hypertension 2018;72:24-43. doi: 10 . 1161/HYPERTENSIONAHA.117.10803

44. National Institute for Health and Care Excellence (NICE). Hypertension in pregnancy: diagnosis and management (NG133). NICE; 2019.

45. Singer M, Deutschman CS, Seymour CW, Shankar-Hari M, Annane D, Bauer M, et al. The third international consensus definitions for sepsis and septic shock (sepsis 3). JAMA 2016; 315:801-10. doi: 10.1001/jama.2016.0287

46. Office for National Statistics. Births by parents' characteristics in England and Wales: 2016. Accessed Oct 8, 2020. https:// www.ons.gov.uk/peoplepopulationandcommunity/birthsdeath- sandmarriages/livebirths/bulletins/birthsbyparentscharacteristicsinenglandandwales/2016

47. Knight M, Kurinczuk JJ, Spark P, Brocklehurst P. Extreme obesity in pregnancy in the United Kingdom. Obstet Gynecol 2010;115:989-97. 10.1097/AOG.0b013e3181da8f09

48. Office for National Statistics. Ethnicity and national identity in England and Wales: 2011. Accessed Oct 8, 2020. https://www. ons.gov.uk/peoplepopulationandcommunity/culturalidentity/ ethnicity/articles/ethnicityandnationalidentityinenglandandwales/2012-12-11

\section{Authors' Data Sharing Statement}

Will individual participant data be available (including data dictionaries)? Yes.

What data in particular will be shared? Individual participant data that underlie the results reported in this article, after deidentification (text, tables, figures, and appendices).

What other documents will be available? Study protocol, case report forms, standard operating procedures, consent form.

When will data be available (start and end dates)? Data will be available between 3 and 36 months after publication.

By what access criteria will data be shared (including with whom, for what types of analyses, and by what mechanism)? Researchers who present a sound analysis plan for any valid research will apply by contacting the corresponding author. The validity of proposals will be assessed by the Kadoorie Critical Care Research Group Data Access Committee (which comprises independent researchers, clinicians, patient, and public representatives). Data will be provided using the Group's current compliant system.

\section{PEER REVIEW HISTORY}

Received August 3, 2020. Received in revised form October 16, 2020. Accepted October 22, 2020. Peer reviews and author correspondence are available at http://links.lww.com/AOG/C172. 